\title{
Massive Chronic Organizing Thyroid Abscess Masquerading Thyroid Cancer
}

\author{
Sudhi Agarwala, Manoj Jain ${ }^{a}$, Gaurav Agarwala, b
}

\begin{abstract}
We present here a case of a 65-year-old male with atypical presentation of long standing chronic thyroid abscess, which initially mimicked malignancy, with a brief discussion of the presentation, diagnosis and possible risk factors for chronic thyroid abscess confined to unilateral thyroid lobe.
\end{abstract}

Keywords: Chronic thyroid abscess; Nodular goiter; Malignancy

\section{Case Report}

A 65-year-old male, hailing from an endemic iodine deficient rural area, presented with history of painless, slowly progressive, euthyroid, non-compressive thyroid swelling starting from the right side of neck for almost 45 years. He did not have any previous history suggestive of thyroiditis, trauma, upper respiratory tract infection, tuberculosis and filariasis. Even with the massive swelling, the patient was asymptomatic, and it was development of a painless ulcer in the dependant part of the massive swelling about 10 days ago, resulting in continuous sero-sanguinous discharge that prompted him to seek treatment. He had never been febrile during the recent course of disease. On examination, the pa-

Manuscript accepted for publication March 25, 2011

\footnotetext{
${ }^{a}$ Departments of Endocrine and Breast Surgery; and Pathology, Sanjay Gandhi Postgraduate Institute of Medical Sciences, Lucknow, India

${ }^{\mathrm{b}}$ Corresponding author: Gaurav Agarwal, Department of Endocrine and Breast Surgery, SGPGIMS, Raebareli Road, Lucknow-226014, India. Email: gaurav@sgpgi.ac.in
}

doi: $10.4021 /$ jem $8 \mathrm{w}$ tient was afebrile, euthyroid and had a huge pendulous thyroid swelling arising from right lobe, extending just short of the nipples in erect posture, with variable consistency and an ulcer at the dependent portion (Fig. 1). Left thyroid lobe was just palpable with no cervical lymphadenopathy. Ultrasound neck and CECT neck showed a heterogeneous lesion with calcification and cystic necrosis (Fig. 2). An ultrasound guided fine needle aspiration cytology from the solid portion of the mass, and cytology of the cyst fluid were done. The cyst fluid was also subjected to bacteriological, fungal and myco-bacterial studies. The fluid cytology revealed plenty of pus cells with occasional gram positives in pairs and short chains. Bacterial culture grew Enterobacter cloacae and Staphylococcus aureus. The hemogram, Serum free-Thyroxin (fT4) and Thyroid Stimulated Hormone (TSH) were within normal limits.

Under ultrasound guidance, a $12 \mathrm{~F}$ Mallecot catheter was inserted in the cystic cavity with drainage of approximately $150 \mathrm{ml}$ of serous fluid, which reduced the size considerably. Under appropriate antibiotic cover, right hemithyroidectomy was done, with excision of a large redundant skin ellipse and neck muscles, and identification and preservation of the external branch superior laryngeal and recurrent laryngeal nerves, and the superior and inferior parathyroids. On naked eye examination, the surgical specimen revealed a normal

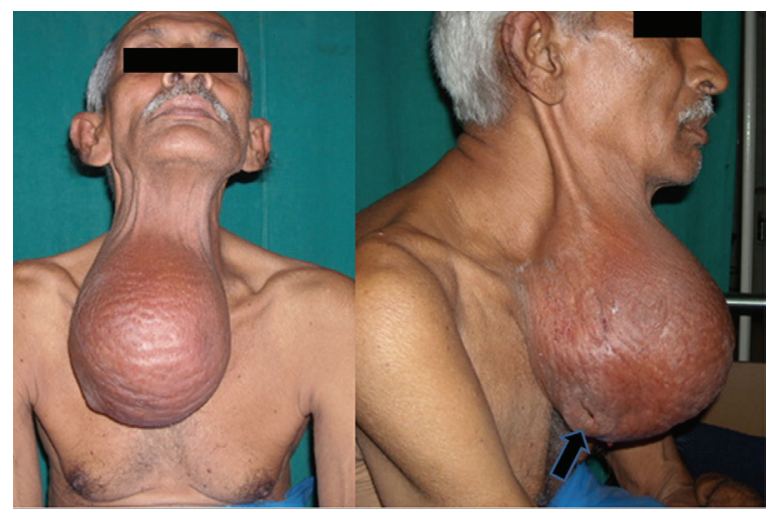

Figure 1. Pendulous goiter reaching to just above nipples, with skin edema and ulcer (arrow). 


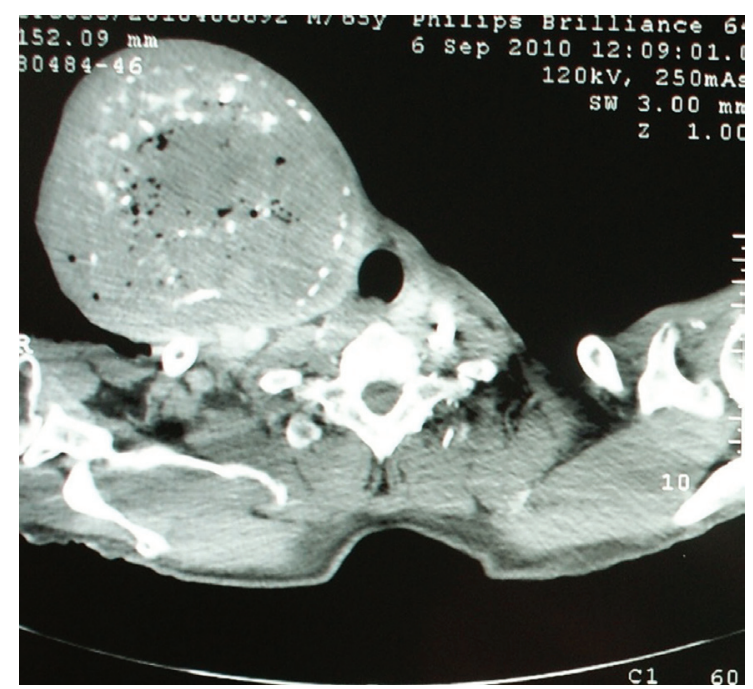

Figure 2. CE-CT Neck showing huge right thyroid nodule with heterogeneous enhancement, areas of cystic degeneration and calcification.

thyroid at periphery, and on histology it showed large areas of necrosis, fibrinoid material, fibro-collagenous tissues and inflammatory infiltrates comprising of neutrophils and lymphocytes (Fig. 3). There were foci of calcification, dilated follicles with flattened epithelium at periphery and no evidence of malignancy. The histological diagnosis was that of chronic organizing abscess in thyroid. Postoperative period was uneventful and patient was discharged after drain removal on the 4th postoperative day free of any complications of surgery. The patient is on regular follow up with no complaints.

\section{Discussion}

Suppurative thyroiditis, though common in pre-antibiotic era, is now rare and mainly reported from developing countries. The suppuration of thyroid gland is mostly a secondary phenomenon with primary infective focus elsewhere, commonest being the upper respiratory tract. The thyroid is relatively protected from infection by its capsule, the rich vascular and lymphatic supply, and its high iodine content [1]. However, various factors like congenital pyriform sinus [2], thyroid/neck injury, blood-borne infections, surrounding infections and intrinsic gland pathology like nodular goitre, thyroid cyst, and papillary thyroid cancer may predispose to suppurative thyroiditis $[3,4]$. Infection in pre-existing thyroid pathology may present with localized thyroid abscess. Suppurative thyroiditis usually presents with features of acute inflammation, however, in patients with immuno-

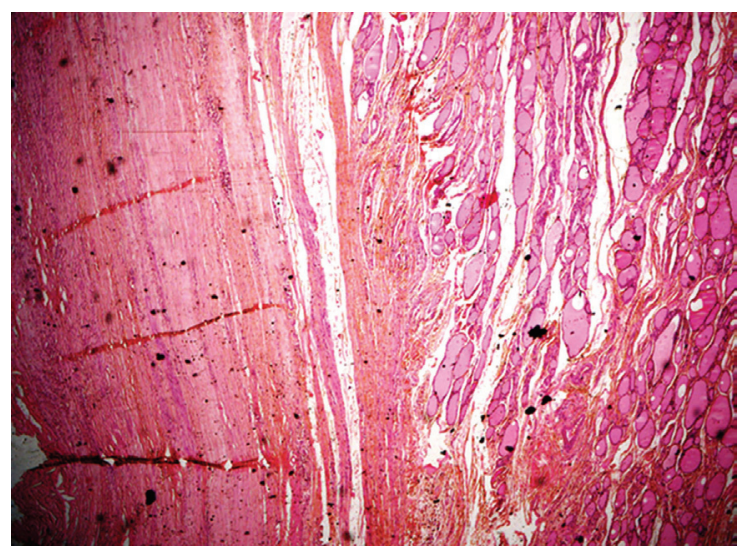

Figure 3. Photomicrograph of section from thyroid nodule showing thyroid follicles and abscess cavity filled with necrotic material and fibrosis suggestive of chronic organizing abscess (H\&E stain, $40 \times$ original magnification).

suppression due to cortico-steroid therapy, diabetes, HIV, old age, or those receiving prolonged or inadequate antibiotic therapy, the acute features of inflammation may not be apparent. The most common organisms isolated are the staphylococcus aureus, other organisms being Escherichia coli, usually following urinary tract infection, mycobacteria, fungi, nocardia and actinomycosis $[4,5]$. It seems highly plausible that the present patient had a pre-existing endemic goiter with thyroid nodule or cyst, with secondary sub-acute infection that resulted in massive thyroid enlargement over a long period of time. A chronic organizing abscess restricted to one thyroid lobe and resulting in such a large swelling without any major symptoms is very rarely seen.

\section{References}

1. Lavini C, Natali P, Magni G, Valli R, Morandi U. Thyroid abscess associated with a substernal goiter. Case report. J Cardiovasc Surg (Torino) 2003;44(2):271-273.

2. Rohondia OS, Koti RS, Majumdar PP, Vijaykumar T, Bapat RD. Thyroid abscess. J Postgrad Med 1995;41(2):52-54.

3. Ameh EA, Sabo SY, Nmadu PT. The risk of infective thyroiditis in nodular goitres. East Afr Med J 1998;75(7):425-427.

4. De Sousa RF, Dilip A, Mervyn C. Thyroid abscess with cutaneous fistula: case report and review of the literature. Thyroid sciences 2008;3(11):CR1-4.

5. Arfeen S, Boast MT, Large DM. Unilateral thyroid swelling due to actinomycosis. Postgrad Med J 1986;62(731):847-848. 